\title{
Polymorphisms in the UGT1A1 gene predict adverse effects of irinotecan in the treatment of gynecologic cancer in Japanese patients
}

This article has been corrected since Advance Online Publication, and an erratum is also printed in this issue.

\author{
Akira Hirasawa $^{1,2}$, Takeru Zama ${ }^{3}$, Tomoko Akahane ${ }^{1}$, Hiroyuki Nomura ${ }^{1}$, Fumio Kataoka ${ }^{1}$, Koichiro Saito ${ }^{3}$, \\ Keisuke Okubo ${ }^{4}$, Eiichiro Tominaga ${ }^{1}$, Kazuya Makita ${ }^{1}$, Nobuyuki Susumu ${ }^{1}$, Kenjiro Kosaki ${ }^{2}$, \\ Yusuke Tanigawara ${ }^{5}$ and Daisuke Aoki ${ }^{1}$
}

Irinotecan is a key chemotherapeutic drug used to treat many tumors, including cervical and ovarian cancers; however, irinotecan can cause toxicity, particularly in the presence of uridine diphosphate glucuronosyltransferase 1A1 (UGT1A1) gene polymorphisms, which are associated with reduced enzyme activity. Here, we investigated the prevalence of three different variants of UGT1A1 (UGT1A1*6, UGT1A1*27 and UGT1A1*28) and their relationships with irinotecan-induced adverse events in patients with gynecologic cancer, who are treated with lower doses of irinotecan than patients with other types of solid tumors. Fifty-three female patients treated with irinotecan and 362 female patients not treated with irinotecan were screened for UGT1A1*6, UGT1A1*27 and UGT1A1*28. Homozygosity for UGT1A1*6 or heterozygosity for UGT1A1*6/*28 was associated with a high risk of severe absolute neutrophil count decrease or diarrhea (odds ratios: 16.03 and 31.33 , respectively). In contrast, serum bilirubin levels were not associated with irinotecan toxicity. Homozygosity for UGT1A1 ${ }^{*} 6 /{ }^{*} 6$ and heterozygosity for $U G T 1 A 1 * 6 /{ }^{*} 28$ were associated with an increased risk of absolute neutrophil count and/or diarrhea in Japanese gynecologic cancer patients, despite the lower doses of irinotecan used in these patients. UGT1A1*6 and UGT1A1*28 are potential predictors of severe absolute neutrophil decrease and diarrhea caused by low-dose irinotecan in gynecologic cancer patients.

Journal of Human Genetics (2013) 58, 794-798; doi:10.1038/jhg.2013.105; published online 3 October 2013

Keywords: gynecologic cancer; hyperbilirubinemia; irinotecan; pharmacogenomics; polymorphism; UGT1A1

\section{INTRODUCTION}

Irinotecan is a key drug used to treat cervical and ovarian cancers; however, $\sim 20-35 \%$ of patients treated with irinotecan experience severe diarrhea and neutropenia. ${ }^{1-5}$ As a prodrug, irinotecan is activated by hydrolysis to SN-38, a potent topoisomerase I inhibitor, and inactivated through conversion to $\mathrm{SN}-38$ glucuronide (SN-38G) by UDP-glucuronosyltransferase $1 \mathrm{~A} 1$ (UGT1A1). ${ }^{6}$ Gene transcription efficiency is inversely correlated with the UGT1A1 gene polymorphism UGT1A1*28.7 Moreover, as UGT1A1 catalyzes bilirubin glucuronidation, homozygosity for the TA7 allele is associated with Gilbert's syndrome, a common mild hyperbilirubinemia. ${ }^{8,9}$ Three variants $\left(U G T 1 A 1^{\star} 6, U G T 1 A 1^{*} 27\right.$ and $\left.U G T 1 A 1^{*} 28\right)$ are associated with reduced enzyme activity and increased irinotecan toxicity, ${ }^{10}$ and the frequencies of these variants differ between Asian and Caucasian populations. ${ }^{10-13}$ These polymorphisms directly reduce UGT enzyme activity. ${ }^{14} U G T 1 A 1^{*} 6$ also can cause a decrease in metabolic activity and is not linked with $U G T 1 A 1^{*} 28 .^{15-17}$

The U.S. Food and Drug Administration recommends that a dose reduction of irinotecan should be considered in patients homozygous for $U G T 1 A 1^{\star} 28$. Furthermore, because of reported ethnic differences, homozygous $U G T 1 A 1^{*} 6 /^{*} 6$ and $U G T 1 A 1^{*} 28{ }^{*} 28$ genotypes and the heterozygous $U G T 1 A 1^{*} 6{ }^{*} 28$ genotype are seen as poor metabolizers (PMs) of irinotecan. Indeed, patients with UGT1A1 deficiencies experience irinotecan-dependent neutropenia and diarrhea in a dose-dependent manner; ${ }^{18}$ however, contradictory results have been reported for the association between UGT1A1 genotypes and toxicity profiles in patients receiving low-dose irinotecan. ${ }^{19-21}$ Moreover, while some studies have suggested its association with severe neutropenia, total bilirubin is yet to be validated as a marker for predicting the risk of severe neutropenia in gynecological cancer

${ }^{1}$ Department of Obstetrics and Gynecology, School of Medicine, Keio University, Tokyo, Japan; ${ }^{2}$ Center for Medical Genetics, School of Medicine, Keio University, Tokyo, Japan; ${ }^{3}$ Department of Otolaryngology-Head and Neck Surgery, School of Medicine, Keio University, Tokyo, Japan; ${ }^{4}$ Department of Otolaryngology, Sano Kosei General Hospital, Tochigi, Japan and ${ }^{5}$ Department of Clinical Pharmacokinetics and Pharmacodynamics, School of Medicine, Keio University, Tokyo, Japan 
patients treated with irinotecan in the absence of UGT1A1 genetic information. ${ }^{22-25}$

To date, only a few studies have described individualized chemotherapy treatment based on the assessment of genetic polymorphisms in gynecologic cancer or on the administration of lower doses of irinotecan for the treatment of gynecologic cancer compared with the doses used for other solid tumors. ${ }^{19}$ Here, we retrospectively investigated the associations of UGT1A1 polymorphisms with serum bilirubin levels and severe adverse effects in Japanese patients who received irinotecan for the treatment of gynecologic cancer.

\section{PATIENTS AND METHODS}

\section{Patients}

Blood samples were collected from 415 Japanese patients who visited the menopausal clinic or cancer follow-up clinic of the Department of Obstetrics and Gynecology, Keio University Hospital (Tokyo, Japan). Fifty-three patients who had cervical or ovarian cancer and received irinotecan-containing chemotherapy were retrospectively studied. An additional 362 patients who did not receive irinotecan therapy were chosen at random. Patients with pathological hyperbilirubinemia, including obstructive jaundice, and nonJapanese patients were excluded from the study. Informed consent was obtained from all patients before using their blood. The study was conducted with the approval of the ethics committee of the School of Medicine, Keio University (approval numbers: 20050135, 20060091 and 20070081).

\section{UGT1A1 genotyping and classification}

Genomic DNA was extracted by standard procedures. UGT1A1*6 $(211 \mathrm{G} \rightarrow \mathrm{A})$, $U_{G T 1 A 1}{ }^{*} 27 \quad(686 \mathrm{C} \rightarrow \mathrm{A})$ and $U_{G T 1 A 1}{ }^{*} 28 \quad\left[(\mathrm{TA})_{6} \mathrm{TAA} \rightarrow(\mathrm{TA})_{7} \mathrm{TAA}\right]$ polymorphisms were genotyped using an Invader UGT1A1 Molecular Assay kit (Third Wave Technologies, Madison, WI, USA) as previously described. ${ }^{26}$ UGT1A1 polymorphisms were classified into two groups, as recommended by the Pharmaceuticals and Medical Devices Agency, Japan (PMDA): the PM group and the extensive metabolizer (EM) group. The PM group included homozygous UGT1A1 ${ }^{*} 61^{*} 6$ and $U G T 1 A 1^{*} 281^{*} 28$ genotypes and the heterozygous $U G T 1 A 1^{*} 61^{*} 28$ genotype. Patients who were not classified as PMs were assigned to the EM group.

Schedule of irinotecan treatment and evaluation of adverse events The treatment regimen consisted of either $60 \mathrm{mg} \mathrm{m}^{-2}$ irinotecan on days 1,8 and 15 and $60 \mathrm{mg} \mathrm{m}^{-2}$ cisplatin on day 1 every 4 weeks or $100 \mathrm{mg} \mathrm{m}^{-2}$ irinotecan alone on days 1,8 and 15 every 4 weeks. Physical examinations and blood tests were retrospectively reviewed to evaluate toxicity and adverse events in irinotecan-treated patients, and their grades were assessed prospectively according to the common terminology criteria for adverse events, version 3.0.27 The monitoring period for adverse events and the worst grade after irinotecan therapy were evaluated until 6 weeks after last irinotecan administration.

\section{Association of irinotecan toxicity with serum bilirubin and UGT1A1 genotypes}

Serum total bilirubin was measured for 392 patients, including 49 patients treated with irinotecan. The highest total bilirubin value was adopted from the patient's own personal historical data in the hospital. Bilirubin measurement was conducted prior to surgery or any chemotherapy in patients given irinotecan, because the bilirubin level may increase after irinotecan therapy. For patients in whom the total bilirubin levels were $1.4 \mathrm{mg} \mathrm{dl}^{-1}$ or greater, hyperbilirubinemia was diagnosed according to the normal range in the hospital. The relationships between serum bilirubin and UGT1A1 genotypes and between serum bilirubin and irinotecan toxicity were statistically analyzed.

\section{Statistical analyses}

Statistical analysis was performed with Exel2011, Microsoft Office: mac2011 (Microsoft Japan Co., Ltd., Tokyo, Japan) with the add-in software Statcel 3 (OMS, Saitama, Japan) and Prism 6 (GraphPad Software, Inc., La Jolla, CA, USA) using the indicated tests. The F-test was used to verify the heterogeneity of variances. Nonparametrically distributed variables were assessed using the Mann-Whitney $U$-test. The chi-square test or Fisher's exact test was performed for contingency tables. Results with $P$-values of less than 0.05 were considered significant.

\section{RESULTS}

\section{Patient characteristics}

Blood samples from a total of 415 patients, including 11 cervical cancer patients, 107 ovarian cancer patients, 225 endometrial cancer patients, 26 ovarian and endometrial double cancer patients, 8 myoma or endometriotic cyst and 38 menopausal disorder were analyzed for UGT1A1 polymorphisms (Table 1). Among these patients, 53 were treated with irinotecan.

\section{UGT1A1 genotype and allele frequency}

Genotype and allele frequencies of UGT1A ${ }^{\star} 6, U G T 1 A 1^{*} 27$ and $U G T 1 A 1^{*} 28$ in 415 patients, including 53 patients that received irinotecan and 362 without irinotecan treatment, are summarized in Supplementary Table 1 .

Two patients $(3.8 \%)$ treated with irinotecan were homozygous for $U G T 1 A 1^{\star} 6$, and none were homozygous for $U G T 1 A 1^{\star} 28$. Fourteen patients $(26.4 \%)$ were heterozygous for $U G T 1 A 1^{*} 6$, and 9 patients $(17.0 \%)$ were heterozygous for $U G T 1 A 1^{\star} 28$. One patient was simultaneously heterozygous for $U G T 1 A 1^{*} 6$ and $U G T 1 A 1^{\star} 28$ (data not shown). No irinotecan-treated patients were heterozygous or homozygous for $U G T 1 A 1^{*} 27$.

None were homozygous for $U G T 1 A 1^{\star} 27$, whereas $17(4.1 \%)$ and 7 (1.9\%) patients without irinotecan treatment were homozygous for $U G T 1 A 1^{\star} 6$ and $U G T 1 A 1^{\star} 28$, respectively.

Of all cases, all $U G T 1 A 1^{\star} 27$ carriers also harbored $U G T 1 A 1^{\star} 28$ alleles (data not shown). Moreover, 14 patients $(9.2 \%)$ were simultaneously heterozygous for $U G T 1 A 1^{\star} 6$ and $U G T 1 A 1^{\star} 28$ (data not shown).

$U G T 1 A 1^{\star} 6$ and $U G T 1 A 1^{*} 28$ groups did not differ significantly between patients receiving or not receiving irinotecan $(P=0.802$ and 0.464 , respectively).

The PM and EM groups did not differ significantly between the total patient population and irinotecan-treated patients $(P=0.605$; Supplementary Table 2).

Correlation between the UGT1A1 genotype and adverse effects of irinotecan

Genotypes and adverse event assessments for 53 patients (11 cervical cancer, 40 ovarian cancer and 2 ovarian and endometrial double cancer patients) who received irinotecan are summarized in Table 2. ${ }^{27}$

Table 1 Characteristics of the patients included in this study

\begin{tabular}{lcc}
\hline Characteristics & $\begin{array}{c}\text { No. of } \\
\text { patients }\end{array}$ & $\begin{array}{c}\text { Irinotecan } \\
\text { administration }\end{array}$ \\
\hline Total & 415 & 53 \\
Age, mean (range) & $52(25-82)$ & $48(29-73)$ \\
& F-test & $P=0.55$ \\
Disease & & \\
Cervical cancer & 11 & 11 \\
Ovarian cancer & 107 & 40 \\
Endometrial cancer & 225 & 0 \\
Ovarian and endometrial double cancer & 26 & 0 \\
Myoma or endometriotic cyst & 8 & 0 \\
Menopausal disorder & 38 & \\
\hline
\end{tabular}


Table 2 Adverse events and effects of the UGT1A1 polymorphism in patients administered irinotecan

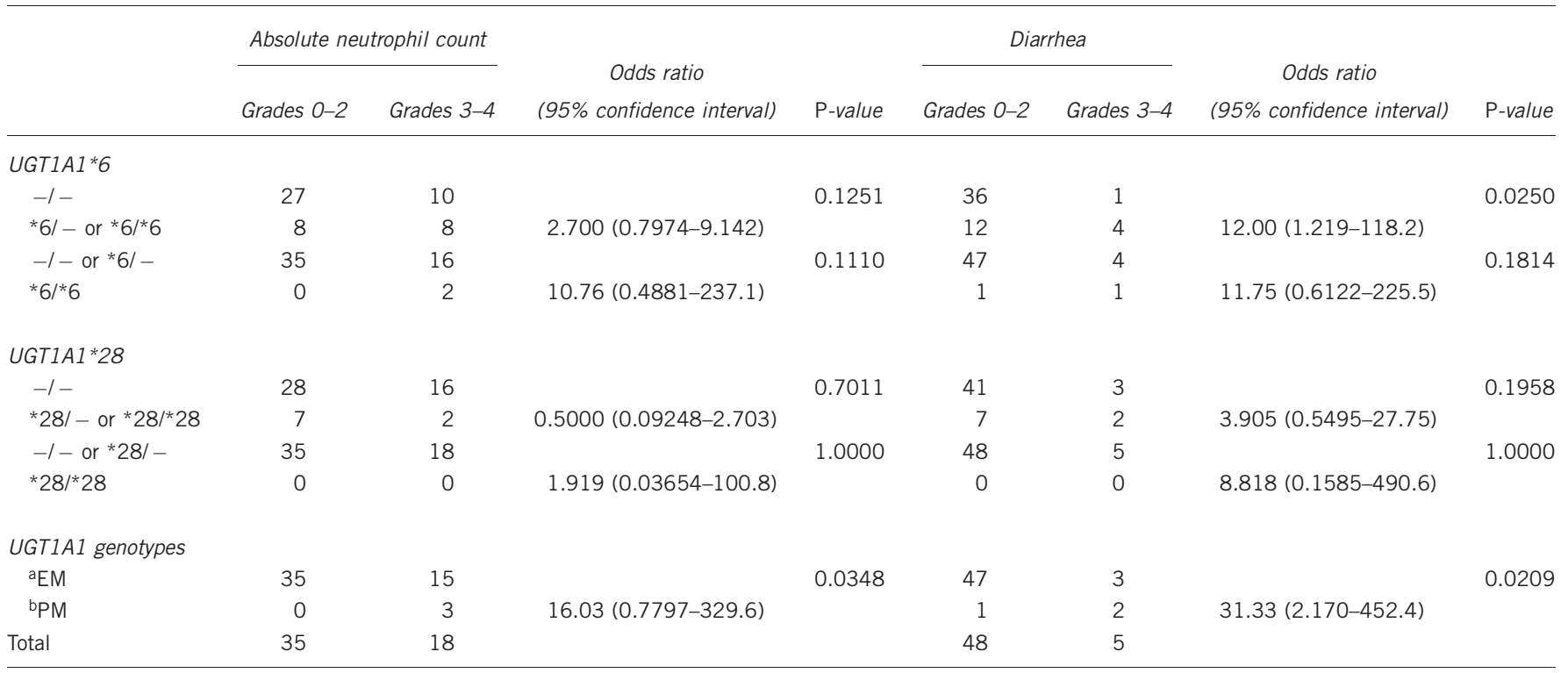

Abbreviations: EM, extensive metabolizer; PM, poor metabolizer.

aOther than PM.

bHomozygous for UGT1A1*6/*6 or UGT1A1*28/*28 or heterozygous for $U G T 1 A 1^{*} 6 /{ }^{*} 28$.

Table 3 Characteristics of three patients classified as PM, who were given irinotecan

\begin{tabular}{|c|c|c|c|c|c|c|c|c|c|}
\hline \multirow[b]{2}{*}{ Patient } & \multirow[b]{2}{*}{ Cancer type } & \multicolumn{3}{|c|}{ UGT1A1 polymorphisms } & \multicolumn{2}{|c|}{ Grade of adverse events ${ }^{a}$} & \multicolumn{3}{|c|}{ Chemotherapy regimen } \\
\hline & & *6 & $* 27$ & *28 & ANC nadir & Diarrhea & $1 s t$ & $2 n d$ & $3 r d$ \\
\hline 1 & Ovarian cancer & ${ }^{*} 6 /{ }^{*} 6$ & $-1-$ & *28/*28 & 3 & 3 & TC & irinotecan & - \\
\hline 2 & Ovarian cancer & $* 6 /-$ & $-1-$ & $* 28 /-$ & 4 & 3 & irinotecan + CDDP & - & - \\
\hline 3 & Ovarian cancer & $* 6 / * 6$ & $-1-$ & *28/*28 & 3 & 1 & $\mathrm{TC}$ & irinotecan + CDDP & DOC \\
\hline
\end{tabular}

Abbreviations: ANC, absolute neutrophil count; CDDP, cisplatin; TC, taxol + carboplatin.

aAdverse events: Common Terminology Criteria for Adverse Events v3.0.

Thirty-five patients had grades 0-2 absolute neutrophil count, whereas 18 patients had grades 3-4 absolute neutrophil count (Table 2). In addition, 48 patients had grades $0-2$ diarrhea, and 5 patients had grades 3-4 diarrhea (Table 2). The occurrence of grades 3-4 absolute neutrophil count and diarrhea was significantly more frequent in PM-group patients than in EM-group patients (odds ratio: $16.03, P=0.0348$ and odds ratio: $31.33, P=0.0209$, respectively). However, $U G T 1 A 1^{\star} 6$ or $U G T 1 A 1^{\star} 28$ alone was not associated with absolute neutrophil count.

Three cases in Table 3 were classified as PMs of irinotecan (the PM group). Two of these cases were homozygous for $U G T 1 A 1^{\star} 6$, and one was simultaneously heterozygous for $U G T 1 A 1^{*} 6$ and $U G T 1 A 1^{*} 28$. All three had a grade 3 or 4 absolute neutrophil count, and two had grade 3 diarrhea.

Relationship between serum bilirubin and UGT1A1 genotypes or irinotecan toxicity

Total bilirubin levels were significantly higher in the PM group $(n=38)$ than in the EM group $(n=354 ; P<0.0001$; Figure 1$)$. Sixtynine out of 392 patients had been diagnosed with hyperbilirubinemia $\left(\geqslant 1.4 \mathrm{mg} \mathrm{dl}^{-1}\right)$ more than once in their history (Table 4$)$. The occurrence of hyperbilirubinemia was significantly increased in heterozygous or homozygous $U G T 1 A 1^{\star} 28$ cases compared with heterozygous or homozygous $U G T 1 A 1^{*} 6$ cases. Furthermore, the occurrence of hyperbilirubinemia was significantly increased in PM- group cases compared with EM-group cases (odds ratio: 6.08, $P<0.0001$; Table 4). However, serum bilirubin levels were not associated with irinotecan toxicity-induced grade 3 or 4 absolute neutrophil count or diarrhea in the 49 patients who were treated with irinotecan $(P>0.05$ for both; Table 5$)$.

\section{DISCUSSION}

Previous studies have revealed ethnic differences in UGT1A1 haplotypes among Caucasians, African-Americans and Asians. ${ }^{7,12-14}$ Conversely, $U G T 1 A 1^{*} 6$ and $U G T 1 A 1^{\star} 27$ polymorphisms have been identified only in Asians. ${ }^{8,13}$ Our results for the allele frequencies of $U G T 1 A 1^{*} 6, U G T 1 A 1^{\star} 27$ and $U G T 1 A 1^{*} 28$ are similar to those of previous reports.

A prior study demonstrates that the $U G T 1 A 1^{\star} 6$ genotype is related to lower SN-38 glucuronidation and a higher frequency of grade $3 / 4$ toxicities in patients receiving irinotecan and cisplatin. ${ }^{28}$ Moreover, a grade $3 / 4$ absolute neutrophil count is significantly more frequent in patients who have the $U G T 1 A 1^{\star} 6$ genotype and are undergoing irinotecan-based chemotherapy, and homozygosity for the $U G T 1 A 1^{*} 6$ allele is associated with altered $\mathrm{SN}-38$ disposition, potentially increasing the risk of severe neutropenia in Asian cancer patients. ${ }^{29}$ Our data could not support this theory, most likely because we did not have enough $U G T 1 A 1^{*} 6$ and $U G T 1 A 1^{*} 28$ cases in this study.

Since $U G T 1 A 1^{\star} 27$ is very rare, its clinical impact has not yet been fully elucidated. The $U G T 1 A 1^{\star} 27$ and $U G T 1 A 1^{*} 28$ alleles are linked; 


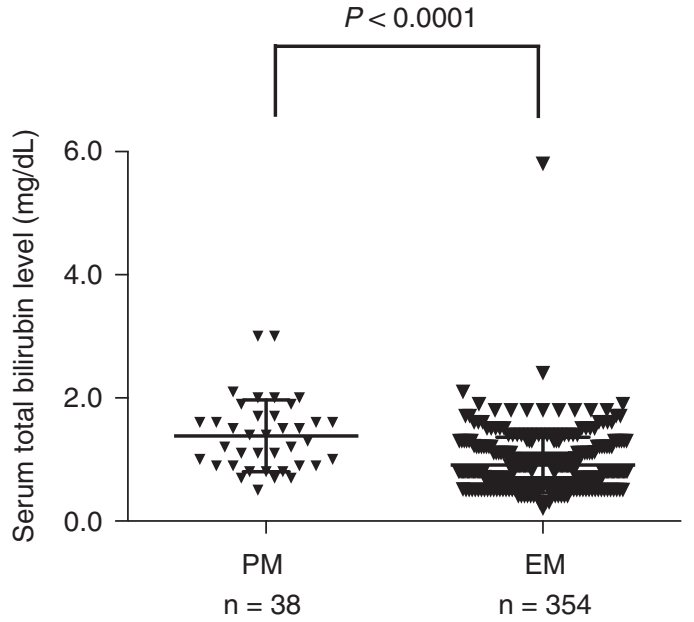

Figure 1 Association between UGT1A1 genotypes and total bilirubin levels. Serum total bilirubin levels were significantly higher in the PM group $(n=38)$ compared with the EM group $(n=354)$, as determined by analysis of 392 patients who had data on serum bilirubin levels $(P<0.0001)$.

Table 4 UGT1A1 genotypes and hyperbilirubinemia

\begin{tabular}{|c|c|c|c|c|}
\hline & \multicolumn{2}{|c|}{ Total bilirubin } & \multirow{2}{*}{$\begin{array}{c}\text { Odds ratio (95\% } \\
\text { confidence interval) }\end{array}$} & \multirow[b]{2}{*}{ P-value } \\
\hline & $<1.4 \mathrm{mgdl}^{-}$ & $4 m g d l^{-1}$ & & \\
\hline \multicolumn{5}{|l|}{$U G T 1 A 1 * 6$} \\
\hline$-1-$ & 221 & 39 & & 0.0576 \\
\hline$* 6 /-$ or $* 6 /{ }^{*} 6$ & 102 & 30 & $1.667(0.9802-2.834)$ & \\
\hline$-1-$ or $* 6 /-$ & 311 & 64 & & 0.1911 \\
\hline$* 6 / * 6$ & 12 & 5 & $2.025(0.6892-5.948)$ & \\
\hline \multicolumn{5}{|l|}{ UGT1A1*28 } \\
\hline$-1-$ & 269 & 38 & & $<0.0001$ \\
\hline${ }^{*} 28 /$ - or & 54 & 31 & $4.064(2.328-7.095)$ & \\
\hline \multicolumn{5}{|l|}{$* 28 / * 28$} \\
\hline$-1-$ or $* 28 /-$ & 321 & 65 & & 0.001 \\
\hline *28/*28 & 2 & 4 & $9.877(1.711-55.08)$ & \\
\hline \multicolumn{5}{|l|}{ UGT1A1 genotypes } \\
\hline apM & 19 & 19 & & $<0.0001$ \\
\hline${ }^{\mathrm{b}} \mathrm{EM}$ & 304 & 50 & $6.080(3.010-12.28)$ & \\
\hline
\end{tabular}

aHomozygous for $U G T 1 A 1^{*} 6 /{ }^{*} 6$ or $U G T 1 A 1^{*} 28 /{ }^{*} 28$ or heterozygous for $U G T 1 A 1^{*} 6 /{ }^{*} 28$. bOther than PM.

therefore, $U G T 1 A 1^{*} 28$ genotyping is thought to cover $U G T 1 A 1^{*} 27$ carriers. ${ }^{30}$ In our study, all four UGT1A1 ${ }^{\star} 27$ carriers also harbored $U G T 1 A 1^{\star} 28$ alleles. Some studies have yielded contradictory results for the relationship between UGT1A1 genotypes and toxicity profiles in patients receiving low-dose irinotecan. ${ }^{19,20}$ For example, the risk of hematologic toxicity is strongly associated with the UGT1A $1^{\star} 28$ genotype at higher doses $\left(>150 \mathrm{mg} \mathrm{m}^{-2}\right)$, but not at lower doses $\left(\leqslant 150 \mathrm{mg} \mathrm{m}^{-2}\right) \cdot{ }^{20}$ However, a recent meta-analysis revealed that the $U G T 1 A 1^{\star} 28 /{ }^{*} 28$ genotype was associated with an increased risk of neutropenia at all doses of irinotecan. ${ }^{21}$

Irinotecan is often administered as combination therapy with cisplatin for gynecologic cancer. In a previous study of 177 Japanese cancer patients treated with irinotecan as a single agent or as combination chemotherapy with cisplatin, ${ }^{31}$ significant associations
Table 5 Associations between serum bilirubin levels and irinotecanrelated adverse events

\begin{tabular}{|c|c|c|c|c|}
\hline \multirow[b]{2}{*}{ Adverse reactions } & \multicolumn{2}{|c|}{ Total bilirubin levels } & \multirow{2}{*}{$\begin{array}{c}\text { Odds ratio (95\% } \\
\text { confidence interval) }\end{array}$} & \multirow[b]{2}{*}{ P-value } \\
\hline & $<1.4 \mathrm{mgdl}^{-1}$ & $\geqslant 1.4 \mathrm{mgdl}^{-1}$ & & \\
\hline \multicolumn{5}{|c|}{ Absolute neutrophil count } \\
\hline Grades $0-2$ & 26 & 6 & & 0.3965 \\
\hline Grades 3-4 & 16 & 1 & $0.2708(0.02979-2.462)$ & \\
\hline \multicolumn{5}{|l|}{ Diarrhea } \\
\hline Grades $0-2$ & 38 & 6 & & 0.5539 \\
\hline Grades 3-4 & 4 & 1 & $1.583(0.1503-16.68)$ & \\
\hline
\end{tabular}

between $U G T 1 A 1^{*} 6$ or $U G T 1 A 1^{*} 28$ and neutropenia were observed for patients in both groups. Moreover, significant associations between $U G T 1 A 1^{\star} 6$ or $U G T 1 A 1^{\star} 28$ and diarrhea were also observed for patients who received irinotecan as a single therapy, but not in patients who received combination therapy with cisplatin. ${ }^{31}$ Of the three PM patients who received irinotecan in our study, one patient was administered irinotecan as a single agent and two patients were given combination chemotherapy with cisplatin (Table 3). Thus, our results demonstrated that significant associations between $U G T 1 A 1^{*} 6$ or $U G T 1 A 1^{*} 28$ and low absolute neutrophil count or diarrhea occurred in Japanese ovarian cancer patients who received irinotecan as single agent or in combination with cisplatin. These findings are in agreement with those of Takano et al., who first reported that grade 3/4 neutropenia, thrombocytopenia and diarrhea were significantly more frequent in $U G T 1 A 1^{*} 6$ and/or $U G T 1 A 1^{*} 28$ genotypes in Japanese gynecologic cancer patients treated with the irinotecan-cisplatin combination. ${ }^{19}$ In contrast, our findings contradicted a previous report showing no significant contribution of UGT1A1 genotypes to toxicity in patients treated with low-dose irinotecan monotherapy. ${ }^{20}$ However, interpatient differences in $\mathrm{SN}$ $38 \mathrm{G}$ formation, which have been correlated with the occurrence of severe diarrhea, and the lower doses of irinotecan used in the present study may, at least in part, explain these conflicting results. ${ }^{32,33}$

Hyperbilirubinemia may be a potential alternative predictive marker for irinotecan toxicity. Indeed, modest elevations in bilirubin (1.0-1.5 $\left.\mathrm{mg} \mathrm{dl}^{-1}\right)$ are associated with increased grades 3-4 neutropenia in metastatic colorectal cancer patients treated with weekly irinotecan. $^{22}$ However, baseline serum bilirubin does not reliably predict overall irinotecan-related toxicity or efficacy in these cases. Interestingly, UGT1A1 polymorphisms are associated with serum bilirubin levels, and $U G T 1 A 1^{*} 28$ has been reported to be associated with Gilbert's syndrome. ${ }^{8,9} \mathrm{UGT1A1}^{\star} 6$, predominant in east Asia, also leads to lower UGT1A1 expression and hyperbilirubinemia. ${ }^{15-17}$ In the current study, hyperbilirubinemia was significantly more frequent in heterozygous or homozygous $U G T 1 A 1^{\star} 28$ cases or PM cases (Table 4), but was not associated with irinotecan toxicity in our study (Table 5). Thus, our findings strongly suggested that UGT1A1 genotyping may be a more powerful predictive biomarker than serum bilirubin.

Our study has some limitations. Because of the sampling procedure used, the patients examined here were cancer survivors, and cases of aggressive disease were not included. In addition, UGT1A1 polymorphisms are thought to influence the development of some cancers, including colorectal, breast and ovarian cancers. ${ }^{34}$ Moreover, an individual's response to irinotecan is unlikely to be affected by UGT1A ${ }^{*} 28$ status. ${ }^{35}$ We also did not distinguish between patients 
receiving irinotecan + cisplatin versus irinotecan alone; this may have affected the outcome of the study. The range of irinotecan dosages in clinical use is very wide and was determined according to organ-based therapies tested in large-scale clinical studies. ${ }^{21}$ Moreover, the metabolism of irinotecan to $\mathrm{SN}-38$ and other metabolites varies widely between patients. Therefore, a wide range of doses is required, and dose modifications or variations should be based on genotyping. Further prospective studies on the pharmacokinetics, pharmacodynamics and pharmacogenetics of irinotecan are needed to clarify these findings, especially in patients receiving low-dose irinotecan, in which few clinical trials have been conducted.

In conclusion, homozygosity for $U G T 1 A 1^{*} 6{ }^{*} 6$ and heterozygosity for both $U G T 1 A 1^{\star} 6 /{ }^{*} 28$ were predictive of low absolute neutrophil count and diarrhea induced by irinotecan in Japanese gynecologic cancer patients, whereas serum bilirubin levels were not.

\section{CONFLICT OF INTEREST}

The authors declare no conflict of interest.

\section{ACKNOWLEDGEMENTS}

This work was supported in part by the Foundation for the Promotion of Cancer Research in Japan (2009-2011). The authors thank the patients for participating in this study. We also thank the assistant medical staff for their help, Mr Hideki Naganishi for his practical support and Ms Keiko Abe and Ms Tomomi Noda for their administrative assistance.

1 Rothenberg, M. L., Kuhn, J. G., Schaaf, L. J., Rodriguez, G. I., Eckhardt, S. G., Villalona-Calero, M. S. et al. Phase I dose-finding and pharmacokinetic trial of irinotecan (CPT-11) administered every two weeks. Ann. Oncol. 12, 1631-1641 (2001).

2 Negoro, S., Fukuoka, M., Masuda, N., Takada, M., Kusunoki, Y., Matsui, K. et al. Phase I study of weekly intravenous infusions of CPT-11, a new derivative of camptothecin, in the treatment of advanced non-small-cell lung cancer. J. Natl Cancer Inst $\mathbf{8 3}$ 1164-1168 (1991).

3 Rothenberg, M. L., Kuhn, J. G., Burris, H. A. 3rd, Nelson, J., Eckhardt, J. R. Tristan-Morales, M. et al. Phase I and pharmacokinetic trial of weekly CPT-11. J. Clin. Oncol. 11, 2194-2204 (1993).

4 Fuchs, C. S., Moore, M. R., Harker, G., Villa, L., Rinaldi, D. \& Hecht, J. R. Phase III comparison of two irinotecan dosing regimens in second-line therapy of metastatic colorectal cancer. J. Clin. Oncol. 21, 807-814 (2003).

5 Vanhoefer, U., Harstrick, A., Achterrath, W., Cao, S., Seeber, S. \& Rustum, Y. M Irinotecan in the treatment of colorectal cancer: clinical overview. J. Clin. Oncol. 19 1501-1518 (2001).

6 Kawato, Y., Aonuma, M., Hirota, Y., Kuga, H. \& Sato, K. Intracellular roles of SN-38, a metabolite of the camptothecin derivative CPT-11, in the antitumor effect of CPT-11. Cancer Res. 51, 4187-4191 (1991).

7 Beutler, E., Gelbart, T. \& Demina, A. Racial variability in the UDP-glucuronosyltransferase 1 (UGT1A1) promoter: a balanced polymorphism for regulation of bilirubin metabolism? Proc. Natl Acad. Sci. USA 95, 8170-8174 (1998).

8 Bosma, P. J., Chowdhury, J. R., Bakker, C., Gantla, S., de Boer, A., Oostra, B. A. et al. The genetic basis of the reduced expression of bilirubin UDP-glucuronosyltransferase 1 in Gilbert's syndrome. N. Engl. J. Med. 333, 1171-1175 (1995).

9 Monaghan, G., Ryan, M., Seddon, R., Hume, R. \& Burchell, B. Genetic variation in bilirubin UPD-glucuronosyltransferase gene promoter and Gilbert's syndrome. Lancet 347, 578-581 (1996).

10 Ando, Y., Saka, H., Ando, M., Sawa, T., Muro, K., Ueoka, H. et al. Polymorphisms of UDP-glucuronosyltransferase gene and irinotecan toxicity: a pharmacogenetic analysis. Cancer Res. 60, 6921-6926 (2000).

11 Ando, Y., Chida, M., Nakayama, K., Saka, H. \& Kamataki, T. The UGT1A1*28 allele is relatively rare in a Japanese population. Pharmacogenetics 8, 357-360 (1998).

12 Hirasawa, A., Akahane, T., Tanigawara, Y. \& Aoki, D. Blood-direct InvaderPlus as a new method for genetic testing. Pers. Med. 9, 657-663 (2012)
13 Fujiwara, Y. \& Minami, H. An overview of the recent progress in irinotecan pharmacogenetics. Pharmacogenomics 11, 391-406 (2010).

14 Ando, Y., Saka, H., Asai, G., Sugiura, S., Shimokata, K. \& Kamataki, T. UGT1A1 genotypes and glucuronidation of $\mathrm{SN}-38$, the active metabolite of irinotecan. Ann Oncol. 9, 845-847 (1998).

15 Jinno, H., Tanaka-Kagawa, T., Hanioka, N., Saeki, M., Ishida, S., Nishimura, T. et al. Glucuronidation of 7-ethyl-10-hydroxycamptothecin (SN-38), an active metabolite of irinotecan (CPT-11), by human UGT1A1 variants, G71R, P229Q, and Y486D. Drug Metab. Dispos. 31, 108-113 (2003).

16 Gagné, J. F., Montminy, V., Belanger, P., Journault, K., Gaucher, G. \& Guillemette, C. Common human UGT1A polymorphisms and the altered metabolism of irinotecan active metabolite 7-ethyl-10-hydroxycamptothecin (SN-38). Mol. Pharmacol. 62 , 608-617 (2002)

17 Yamamoto, K., Sato, H., Fujiyama, Y., Doida, Y. \& Bamba, T. Contribution of two missense mutations (G71R and Y486D) of the bilirubin UDP glycosyltransferase (UGT1A1) gene to phenotypes of Gilbert's syndrome and Crigler-Najjar syndrome type II. Biochim. Biophys. Acta 1406, 267-273 (1998).

18 Marsh, S. \& Hoskins, J. M. Irinotecan pharmacogenomics. Pharmacogenomics 11 1003-1010 (2010)

19 Takano, M., Kato, M., Yoshikawa, T., Sasaki, N., Hirata, J., Furuya, K. et al. Clinical significance of UDP-glucuronosyltransferase $1 A 1 * 6$ for toxicities of combination chemotherapy with irinotecan and cisplatin in gynecologic cancers: a prospective multi-institutional study. Oncology 76, 315-321 (2009).

20 Hoskins, J. M., Goldberg, R. M., Qu, P., Ibrahim, J. G. \& McLeod, H. L. UGT1A1*28 genotype and irinotecan-induced neutropenia: dose matters. J. Natl Cancer Inst. 99, 1290-1295 (2007)

21 Hu, Z. Y., Yu, Q., Pei, Q. \& Guo, C. Dose-dependent association between UGT1A1*28 genotype and irinotecan-induced neutropenia: low doses also increase risk. Clin. Cancer Res. 16, 3832-3842 (2010).

22 Meyerhardt, J. A., Kwok, A., Ratain, M. J., McGovren, J. P. \& Fuchs, C. S. Relationship of baseline serum bilirubin to efficacy and toxicity of single-agent irinotecan in patients with metastatic colorectal cancer. J. Clin. Oncol. 22, 1439-1446 (2004).

23 Innocenti, F., Undevia, S. D., Iyer, L., Chen, P. X., Das, S., Kocherginsky, M. et al. Genetic variants in the UDP-glucuronosyltransferase $1 \mathrm{~A} 1$ gene predict the risk of severe neutropenia of irinotecan. J. Clin. Oncol. 22, 1382-1388 (2004).

24 Ramchandani, R. P., Wang, Y., Booth, B. P., Ibrahim, A., Johnson, J. R., Rahman, A et al. The role of $\mathrm{SN}-38$ exposure, UGT1A1*28 polymorphism, and baseline bilirubin level in predicting severe irinotecan toxicity. J. Clin. Pharmacol. 47, 78-86 (2007).

25 Freyer, G., Rougier, P., Bugat, R., Droz, J. P., Marty, M., Bleiberg, H. et al. Prognostic factors for tumour response, progression-free survival and toxicity in metastatic colorectal cancer patients given irinotecan (CPT-11) as second-line chemotherapy after 5FU failure: CPT-11 F205, F220, F221 and V222 study groups. Br. J. Cancer 83, 431-437 (2000).

26 Hasegawa, Y., Sarashina, T., Ando, M., Kitagawa, C., Mori, A., Yoneyama, M. et al. Rapid detection of UGT1A1 gene polymorphisms by newly developed Invader assay. Clin. Chem. 50, 1479-1480 (2004).

27 Common terminology criteria for adverse events, version 3.0, 2006. [Cited 23 June 2013.] Available at: (http://ctep.cancer.gov/reporting/ctc_v30.html).

28 Han, J. Y., Lim, H. S., Shin, E. S., Yoo, Y. K., Park, Y. H., Lee, J. E. et al. Comprehensive analysis of UGT1A polymorphisms predictive for pharmacokinetics and treatment outcome in patients with non-small-cell lung cancer treated with irinotecan and cisplatin. J. Clin. Oncol. 24, 2237-2244 (2006).

29 Jada, S. R., Lim, R., Wong, C. I., Shu, X., Lee, S. C., Zhou, Q. et al. Role of UGT1A1*6, UGT1A1*28 and ABCG2 c.421C >A polymorphisms in irinotecan-induced neutropenia in Asian cancer patients. Cancer Sci. 98, 1461-1467 (2007).

30 Sai, K., Saeki, M., Saito, Y., Ozawa, S., Katori, N., Jinno, H. et al. UGT1A1 haplotypes associated with reduced glucuronidation and increased serum bilirubin in irinotecanadministered Japanese patients with cancer. Clin. Pharmacol. Ther. 75, 501-515 (2004).

31 Minami, H., Sai, K., Saeki, M., Saito, Y., Ozawa, S., Suzuki, K. et al. Irinotecan pharmacokinetics/pharmacodynamics and UGT1A genetic polymorphisms in Japanese: roles of UGT1A1*6 and *28. Pharmacogenet. Genomics 17, 497-504 (2007)

32 Gupta, E., Lestingi, T. M., Mick, R., Ramirez, J., Vokes, E. E. \& Ratain, M. J. Metabolic fate of irinotecan in humans: correlation of glucuronidation with diarrhea. Cancer Res. 54, 3723-3725 (1994).

33 Gupta, E., Mick, R., Ramirez, J., Wang, X., Lestingi, T. M., Vokes, E. E. et al. Pharmacokinetic and pharmacodynamic evaluation of the topoisomerase inhibitor irinotecan in cancer patients. J. Clin. Oncol. 15, 1502-1510 (1997).

34 Strassburg, C. P. Pharmacogenetics of Gilbert's syndrome. Pharmacogenomics 9 , 703-715 (2008)

35 Dias, M. M., McKinnon, R. A. \& Sorich, M. J. Impact of the UGT1A1*28 allele on response to irinotecan: a systematic review and meta-analysis. Pharmacogenomics 13, 889-899 (2012)

Supplementary Information accompanies the paper on Journal of Human Genetics website (http://www.nature.com/jhg) 